\title{
Modeling and Experiments in Cell and Biomolecular Mechanics
}

\author{
G. Bao • A. Vaziri • H.D. Espinosa
}

Published online: 10 January 2009

(C) Society for Experimental Mechanics 2009

With recent advances in cell and molecular biology, biomechanics and bioengineering, many researchers in the mechanics and materials field are performing experimental and modeling studies of cell and biomolecular mechanics. The behavior of cells and tissues as complex biological systems is a result of integrated and regulated interactions among many components such as cell cytoskeleton, extracellular matrix (ECM), signal transduction pathways, intracellular secretion/transport, and gene expression. Mechanical forces and deformations may play an important role in all these aspects, and in regulating cell behavior and function. To understand these important issues, extensive studies have been performed to develop new experimental and modeling approaches, including multi-scale modeling, optical and magnetic tweezers, AFM, micropipette, and MEMS/NEMS devices for single cell and single molecule mechanics testing. These recent advances have stimulated, and benefited from, the development of engineered materials for biological applications. This special issue of Experimental Mechanics on Modeling and Experiments in Cell and Biomolecular Mechanics will illustrate some of the

G. Bao $(\bowtie$, SEM Member)

Department of Biomedical Engineering,

Georgia Institute of Technology and Emory University,

Atlanta, GA, USA

e-mail: gang.bao@bme.gatech.edu

\section{A. Vaziri}

Department of Mechanical and Industrial Engineering,

Northeastern University,

Boston, MA, USA

e-mail: vaziri@coe.neu.edu

H.D. Espinosa (SEM Member)

Department of Mechanical Engineering, Northwestern University,

Evanston, IL, USA

e-mail: espinosa@northwestern.edu recent developments in cell and biomolecular mechanics, aiming to stimulate further development of this exciting field.

In this special issue, we have assembled a number of recent studies in the field of mechanics of living cells and biomolecules, ranging from detailed hierarchical atomistic simulations of proteins to multiscale computational models for mechanics of encapsulated cells. Several review articles are also included which highlight the state of the art in this area. A brief overview of each article published in this special issue is given below.

In "Quantifying forces mediated by integral tight junction proteins in cell-cell adhesion," Vedula et al. studied cellular adhesion by measuring the force needed to separate two cells using the dual micropipette assay. This work provides insight into the biomolecular mechanisms of cell adhesion and the effect of various treatments on forces required for cell separation.

In "Dissecting the molecular basis of the mechanics of living cells," Kumar and LeDuc provided a thorough review of technologies that have recently emerged for measuring the structural and functional contributions of subcellular components. The implications of these techniques in deciphering molecular basis of cell structure and mechanics are also highlighted in this review article.

In "Changes in intracellular calcium during compression of C2C12 myotubes," Ceelen et al. investigated the changes to the $\mathrm{Ca}^{2+}$-influx in cells under compression and its correlation with cell behavior and death. This study has implications in understanding the underlying mechanisms of tissue damage at the cellular level.

In "Numerical simulation of nanoindentation and patch clamp experiments on mechanosensitive channels of large conductance in Escherichia coli," Tang et al. provided a multiscale numerical framework for simulating the response of a mechanosensitive channel of large conductance in $E$. 
coli embedded in a vesicle. The developed multiscale model was used to analyze the detailed structural transitions of the protein when the lipid vesicle is subjected to nanoindentation and patch clamp experiments.

In "Stiffening and contraction induced by dexamethasone in alveolar epithelial cells," Puig et al. studied the effects of Dexamethasone on the stiffness and contractility of alveolar epithelial cells using magnetic twisting cytometry and traction microscopy. The results elucidate the cellular mechanisms that affect the structural integrity of the alveolar monolayer during lung inflammation.

In "Regulation of cell adhesion free energy and forces by external sliding velocities," Yang and Zaman investigated the molecular mechanisms of cell adhesion using a novel molecular thermodynamic model. The study provides the free energy landscapes under a broad range of external forces, binding energies and receptor surface coverage, providing significant insight into cell adhesion and the factors affecting this intricate phenomenon.

In "The molecular mechanics of collagen degradation: Implications for human disease," Salsas-Escat and Stultz employed molecular simulations to study the mechanics of collagen degradation. Their study highlights how the dynamical simulations of collagen can provide new opportunities for the development of therapies for disorders that are associated with abnormal collagen catabolism.

In "Molecular Mechanics of Stutter Defects in Vimentin Intermediate Filaments," Ackbarow and Buehler developed an Extended Bell Model to provide a theoretical description of the unfolding behavior of coiled-coil structures. Their results suggest that the stutter represents a molecular defect at which unfolding occurs at lower forces than in the rest of the protein and has direct implication in understanding the mechanics and biological function of vimentin.

In "Computational analysis of a cross-linked actin-like network," Kim et al. developed a three-dimensional model of actin-like networks using Brownian dynamics simulations. The computational model was used to analyze the effects of various system parameters on the growth and morphology of the network. This model provides new avenues for predicting and simulating the actin-related phenomena and therefore has direct implications in understanding the behavior of living cells.
In "The potential of MEMS for advancing experiments and modeling in cell mechanics," Loh et al. provided an overview of the experimental and model-based investigations that have been used for studying cell mechanics and the structure-function relationship that dictate living cells' behavior. In this article, novel MEMS sensor and actuator schemes that have yet to materialize in this field are also discussed.

In "Morphological responses of vascular endothelial cells induced by local stretch transmitted through intercellular junctions," Ueki et al. studied the underlying mechanisms of cell structural remodeling by applying a local tension to the endothelial cells using a glass microneedle. The results illuminate the mechanotransduction pathways at the subcellular level.

In "Microfabricated force sensors and their applications in the study of cell mechanical response," Yang and Saif introduced a class of microfabricated force sensors that can be used for measuring large force response of living cells. The implications of the developed sensors in studying cell mechanical response under large deformations are also discussed.

In "Protein mechanics: A new frontier in biomechanics," Bao provides an overview of the role of protein deformation in force-regulated biological functions of living cells including mechanosensing and mechanotransduction. The experimental and modeling challenges in protein mechanics are also discussed in this article.

These studies and reviews provide broad examples of recent advancements in some of the most important areas of biomechanics and biomaterials, and give new perspectives of further developing the field of cell and molecular biomechanics. Our hope is that the articles presented in this special issue will further stimulate a strong interest in the applied mechanics community to advance the mechanics of biology and medicine.

Finally, we wish to thank the Society for Experimental Mechanics and Springer-the publisher of Experimental Mechanics - for giving us the opportunity to organize this special issue of the journal, all the contributing authors for their excellent papers, as well as the anonymous reviewers who helped immensely in shaping this special issue in the current form. 